\title{
Managed Exercise Monitoring: a Novel Application of Wireless On- Body Inertial Sensing
}

\author{
Lawrence Cheng, Stephen Hailes, Member of IEEE
}

\begin{abstract}
The idea of "Physical Exercises (PEs) homework" has been introduced to encourage children and potential young athletes to stay physically active outside of classroom and to keep up with training. However, it is not practical to assume all parents are willingly and responsible enough to supervise home PE sessions. To turn the concept of managed PE homework into a reality, an automated solution is needed to address the lack of supervision problem. This paper presents a novel managed exercise monitoring application - that uses wireless on-body inertial sensor to record, analyse and assess the quality of the exercises carried out by a subject - to address the supervision problem. By automating the system, parents are neither required to participate with, nor to supervise the child during the entire home PE session. Such arrangements are in placed to improve the practicability of the $\mathrm{PE}$ homework concept.
\end{abstract}

\section{INTRODUCTION}

It is well-known that Physical Exercises (PE) are good for health: this statement is especially true among young children in developed countries, in which governments are struggling to find adequate solutions to fight against children obesity. However, as reported in [1], British children among the 5+ to $11+$ years old are being offered a 30-min session of PE per week. Furthermore, state school students do only half as much PE a week as those in the private sector, largely due to the lack of (human) resources for supervision [1][5]. The lack of supervision resources problem would subsequently hammer the availability of potential young athletes. To make children and potential young athletes exercise more, the idea of "PE homework" [2][3][4], has emerged. PE homework, unlike traditional homework which are usually written assignments that can be checked upon off-line, is a range of physical exercise assignments (such as jogging, aerobic exercises, and more) assigned to children, which they should complete after school hours. The aim of this idea is to encourage children and potential young athletes to remain physically active outside the (limited) school hours. However, children need to be disciplined; but it is not practical to assume all parents have time to participate with the children during their entire home PE sessions. Thus, the fundamental problem, i.e. lack of supervision, must be addressed.

Supervision requires some form of activity recording (to prove the PE assignment was completed); the easiest way is to use a video camera to capture all activities. However, this would require the teachers to check through all the videos (i.e. similar to checking written assignments): this arrangement would contradict to the original purpose of PE homework (as it is not scalable and would simply add more work load to the teachers). Also, it would require subjective assessments to assess the quality of the conducted exercises. Thus, there is a need of an automated solution to enable out-of-classroom PE exercises. By automated solution, we mean the system should be able to monitor a child whist he/she is completing his/her PE homework (after classes), with limited adult intervention. The system should be able to determine when and how long an exercise has been carried out, and should provide also the necessary information to enable supervisors to determine (off-line) whether the exercise was carried out in a proper fashion. By proper fashion, we mean that, say, a running exercise should be carried out at the specified (minimum) speed for the assigned duration. Furthermore, the system should provision supervisors to assess the quality of the exercises conducted by potential young athletes.

There are related works in the area: a set of active or reflective markers can be placed on different segments of a subject, and motion can be tracked using optical-based camera systems. However, this is complicated to setup (requires multiple markers and precisely positioned cameras) and most importantly, monetary expensive. Alternatively, cost-effective pedometers can be used for counting steps [4][9]. However, these devices are generally considered as less accurate [12], which means the approach would not be a suitable solution for assessing the quality of the exercises. Other related systems include wearable heartbeat monitors, which can be found in many wearable devices such as watches; but a (constant) fast heartbeat does not provide any information on whether an exercise has been carried properly. The same applies to sweat monitors [6]: a high(er) sweat rate can refer to a slow walk under the sun in a very hot and humid day.

We believe that, with recent advances in inertial sensing technologies and their increasingly affordable prices, an optimal solution to PE homework can be built based on inertial sensing. Inertial sensing uses inertial sensors (such as accelerometers, gyros, and more) to capture motion information of part(s) of a moving body. The captured motion information can be used to assess the quality of the conducted exercises. In this paper, we shall present and analysis the results of a wireless on-body inertial sensing system developed by the SEnsing for Sports And Managed Exercise (SESAME) project [8][11]. We will demonstrate how the system is capable of enabling managed exercises for children and potential young athletes. 


\section{SySTEM DESIGN}

\section{A. System Assumptions \& Requirements}

We have discussed that the aim of the system is to provide an automated solution to supervise children and potential young athletes to ensure they have completed their PE homework properly (out-of-classroom). When designing our system, we assume that: a) a typical x-story house in a developed country where children can exercise in a safe and spacious environment (i.e. in the backyard); b) domestic 802.11-based wireless coverage -802.11 is assumed because it is the most common type of wireless coverage available in domestic environment; c) we assume PE homework are sprint-related exercises in this paper because they are the most common type of PE assignment that would be assigned to individual child (i.e. no team effort required); d) we further assume that moderate weekly exercises are required (e.g. a few hours per week) thus, efficient power consumption is not addressed in this paper (i.e. batteries are replaceable). Addressing security issues (such as authentication and confidentiality protection) and designing step detection algorithms are outside the scope of this paper. The system should be lightweight and powered by batteries; it should support ondevice (optional) and off-device data storage, with wireless data transmission (i.e. the use of wires should be avoided to minimise chances of injuries caused by motion obstruction). The system should contain wearable inertial sensor(s) that collect necessary types of motion data in order for verifying whether the PE assignment was carried out properly. Ideally, the system should provide near realtime (i.e. immediately after the exercise) reports to the user (i.e. the parent) to encourage the parent to participate at some points during the child's home PE session.

\section{B. System Description}

Figure 1 shows the wireless inertial sensor prototype that we have designed and implemented. We chose the MTx sensor from xSens [10] as our sensing unit for its completeness: an MTx sample represents all essential (i.e. 6 Degree of Freedom) motion related data including acceleration, magnetometer readings, gyros readings, temperature, timestamps, etc ${ }^{1}$. The sensor is connected to a 802.11 wireless interface (i.e. a connectBlue WiFi module OWSPA311g) through a RS232 interface. The sensor is powered by a pack of battery. The assembled unit is slightly larger than the size of a coin, and weighs $\sim 75 \mathrm{~g}$ (including a battery pack). We have verified that our sensor can continuous transmit data wirelessly at a high sampling (data) rate $(120 \mathrm{~Hz})$ for $1.5 \mathrm{hrs}$ using a battery pack.

Figure 2 shows the system design. The wireless inertial sensor (Figure 1) is attached to the back of the subject to collect motion data of the subject during a sprint session. The Home Server is an initial data collection point. Data are sent wirelessly from the sensor to the Home Server, where data processing, such as motion analysis, are carried

${ }^{1}$ Different types of information are needed to compensate noises in data sets: for example the acceleration and magnetic data are often used to correct the orientation data measured by the gyros in order to reduce drift. out. The results of the processed data are stored and later presented to the parent at the Home Server. The computed data can be transferred through the Internet to the School Server, at which the data will be stored and used as a proof of record (that the assigned PE homework was completed). The initial data processing at the Home Server enables the supervisors to determine whether the assigned exercise was carried out properly. For example, using the timestamps, one can determine the duration of the exercise, or more specifically, the duration(s) of which a proper run was being made (see section III). All analysis processes in the current implementations are carried out off-line.

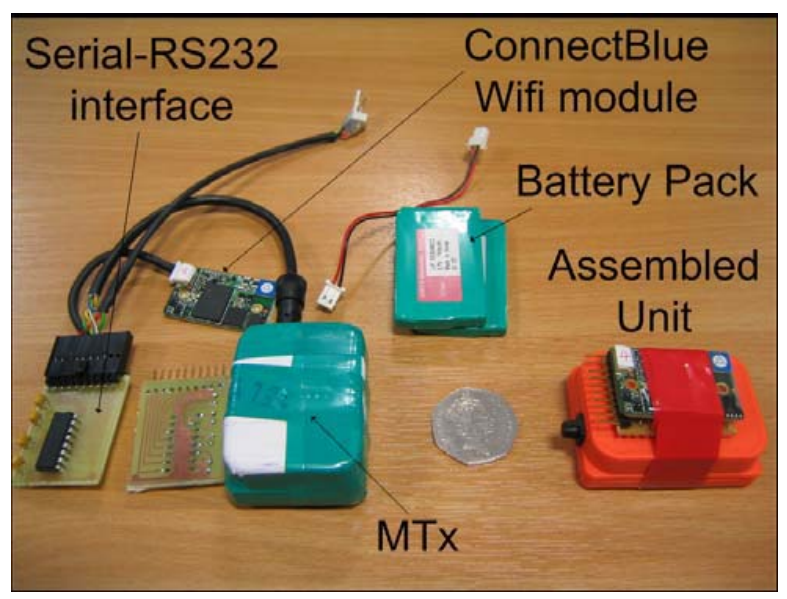

Figure 1 - A wireless inertial sensor

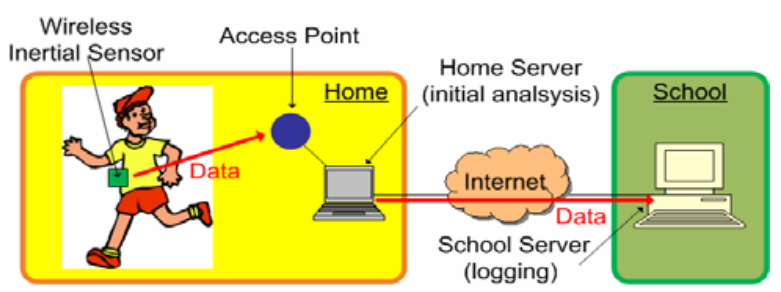

Figure 2 - The system design

Our design does not include sensor node and on-body personal server, which are generally used to initially process raw sample data to reduce data rates [9]. This is because: a) by minimising additional on-body equipment needed, the setup process is simplified to promote psychological acceptance and to minimise chances of injury; furthermore, the additional weight on the subject (i.e. a child) is minimised; b) the scenario is based in an indoor environment covered by wireless connectivity, thus data rate reduction is less important ${ }^{2}$.

\section{System Evaluation}

\section{A. Experiment Setup}

The experiment involves walking and jogging; the former would be considered as improper exercising. Our wireless sensor (Figure 1) is configured to sample raw (i.e. un-calibrated) data at $120 \mathrm{~Hz}$ (i.e. default working sampling

\footnotetext{
${ }^{2}$ Using the MTx sensor as a reference: it can be used to collect 60 -byte samples at a default working rate of $120 \mathrm{~Hz}$. This yields a maximum data rate of $\sim 58 \mathrm{Kbps}$, which can be easily accommodated by any 802.11 based protocol even when multiple subjects are present.
} 
rate of MTx). A (relatively) high sampling rate is used, this is because we would like to capture all motions in detail, which would be useful when analysing the data (see shortly later). All captured data are delivered wirelessly to the Home Server using TCP. The sensor is attached to the lower back of the subject. Note that the sensor collects 3D accelerations: the front-back (i.e. forward) acceleration is $\mathrm{Z}$, the up-down (i.e. vertical) acceleration is $\mathrm{X}$, whereas the horizontal acceleration is Y. Lower-back was chosen because: a) it is the easiest place the hold the sensor firmly and securely (to minimise the effect of noise created by vibrations of soft human tissue); b) it is closet to the CoM of the body, where forces from all directions exerted on the subject can be detected.

\section{B. Analysis Model}

A "step" involves three stages: the $1^{\text {st }}$ swing phase (i.e. the leg swings behind the CoM), the $2^{\text {nd }}$ swing phase (i.e. the leg swings forward of the CoM), and the heel-touchdown phase. Figure $3 \mathrm{a}$ and Figure $3 \mathrm{~b}$ show how theoretically - the vertical and horizontal accelerations would change during a step [7]. Note that, a step should be considered in pair because it involves two foot (i.e. left and right feet).

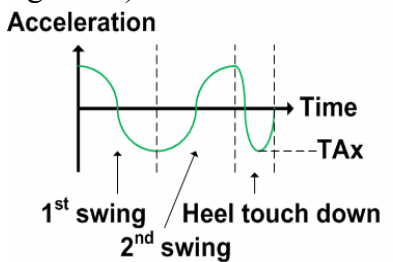

(a) Theoretical vertical acc

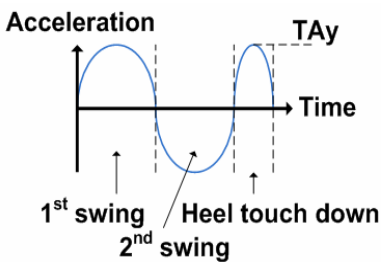

(b) Theoretical horizontal acc
Figure 3 - Theoretical accelerations of walking steps (not to scale)

\section{Results and Analysis}

Figure 4 shows the vertical and horizontal accelerations of 10 walking steps. The heel-touch-down phases (explained in Figure 3b) are clearly shown in Figure 4. Figure 5 shows the differentiated horizontal acceleration signals. Differentiated acceleration data are known as "jerks" [13], and represent the peaks of changes in momentum. By matching the peaks in Figure 5 with the original signals (Figure 4), the step duration is $\sim 2$ second, which would be too slow for a run, hence this part of the exercise would be considered as an improper.

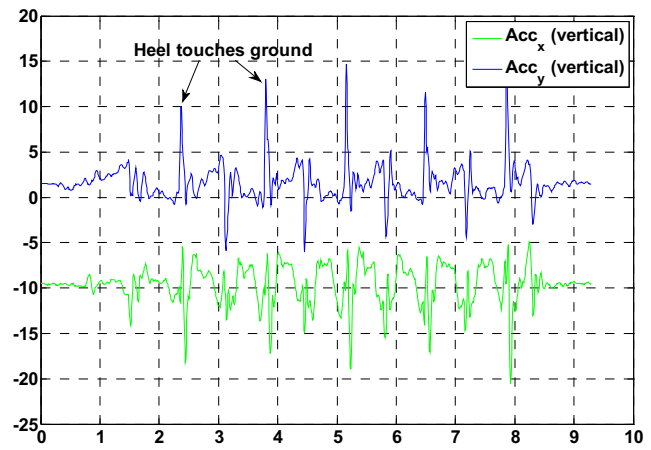

Figure 4 - Vertical and horizontal accelerations of walking steps

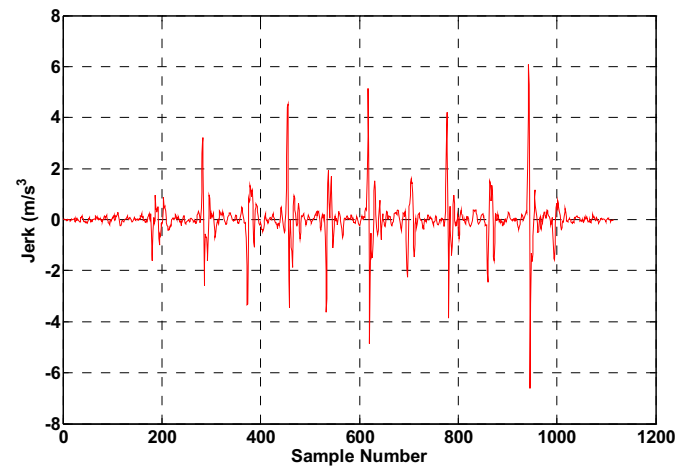

Figure 5 - Differentiated signals of walking steps (horizontal accelerations)

In contrast, Figure 6 and Figure 7 show the vertical and horizontal raw and differentiated acceleration data of jogging steps respectively. Note that, the magnitude of changes in accelerations is much larger during jogging (when comparing Figure 4 and Figure 6). This is due to the increase in impact forces when the heel touches ground during jogging, which involves more rapid movements of the body. Note also that, the step duration is much shorter $(\sim 0.32 \mathrm{sec})$. The first pair of steps made during the jog was ignored as they are below a threshold. The step duration of the steps that are above the threshold are added up to provide a total exercise time.

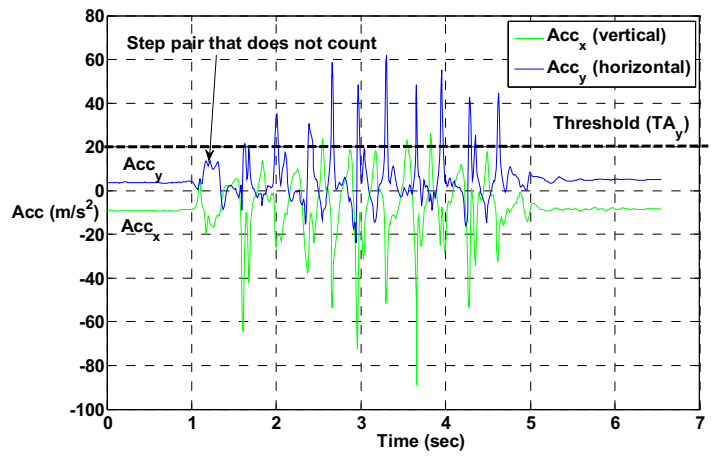

Figure 6 - Vertical and horizontal accelerations of jogging steps

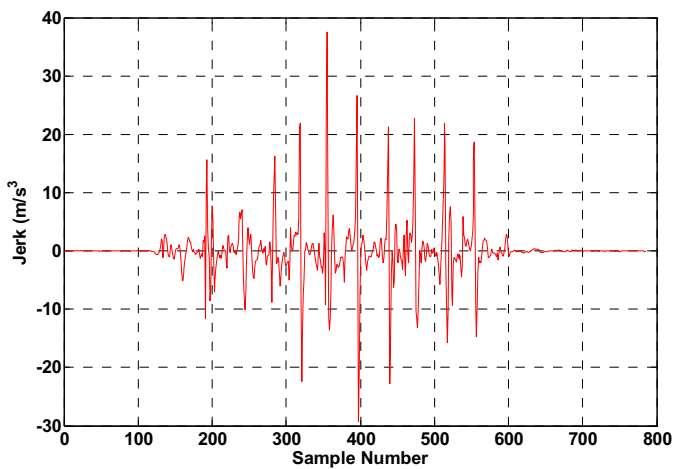

Figure 7 - Differentiated signals of jogging steps (horizontal accelerations) 


\section{CONCLUSION}

In order to enable children and potential young athletes to stay physically active out-of-classroom, the concept of PE homework has emerged. However, due to a lack of available resources for supervision, an automated solution is needed. We have presented a novel body inertial sensing application that enables children and potential young athletes to participate out-of-classroom PE sessions. We have presented our wireless sensor prototype, which uses increasingly affordable COTs products and common domestically-available technologies such as 802.11 to demonstrate how our system is capable of capturing the necessary inertial information of the body for automatically analysing the quality of PE assignments. Through such arrangement, parental guidance is only required at the equipment setup phase, hence addressing the problem of lack of available resource for supervisions. We have presented that the quality of exercises can be monitored, analysed and reported. Our work therefore provides a practical approach to achieving the PE homework concept.

\section{FUTURE WORK}

As part of our future work, we plan to attach multiple inertial sensors to different segments of a subject, to determine whether increasing the number of data sources improves the quality of the analysis.

\section{ACKNOWLEDGEMENTS}

This paper describes work undertaken in the context of the EPSRC-funded SEnsing for Sport And Managed Exercise (SESAME) project (EP/D076943).

\section{REFERENCES}

[1] T. Helm, "UK State School Pupils Lag in Exercise League", Daily Telegraph, $7^{\text {th }}$ Jan 2008 ,

http://www.telegraph.co.uk/news/main.jhtml?xml=/news/2008/01/ 07/nschool107.xml
[2] "Pediatric Obesity: Healthier Tomorrows Helps Kids Get Fit", Department of Pediatrics, North Shore LIJ Huntington Hospital, USA, http://www.hunthosp.org/patientservices/pediatricobesity.html

[3] R. Gabbei, D. Hamrick, "Using Physical Activity Homework to Meet the National Standards", in the Journal of Physical Education, Recreation \& Dance, Vol. 72, 2001.

[4] J. Inchley, L. Cuthbert, M. Grimes, "An Investigation of the use of Pedometers to Promote Physical Activity, and Particularly Walking, Among School-Aged Children", in Report to Paths to Health and the Scottish Health Promoting Schools Unit", May 2007. http://www.pathsforall.org.uk/cms uploads/CARHU2007.pdf

[5] M. Oliver, G. Schofield, G. Kolt, P. Schluter, "Pedometer Accuracy in Physical Activity Assessment of Preschool Children", in Journal of Science and Medicine in Sport, Vol. 10, Issue 5, Oct 2007, pp. 303-310.

[6] F. Francesco, D. Costanzo, P. Salvo, D. Rossi, "Towards the Measurement of Sweat Rate via Wearable Sensors", in Proccedings of Personalised Health, May 2007, Greece.

[7] J. Kim, H. Jang, D. Hwang, C. Park, “A Step, Stride and Heading Determination for the Pedestrian Navigation System", in Journal of Global Positioning Systems 2004, Vol. 3, No. 1-2: 273-279.

[8] The SESAME Project, http://www.sesame.ucl.ac.uk

[9] G. Borriello, W. Brunette, J. Lester, P. Powledge, A. Rea, "An Ecosystem of Platforms to Support Sensors for Personal Fitness", in Proceedings of IEEE International Workshop on Wearable and Implantable Body Sensor Network (BSN), Boston, USA, April 2006.

[10] The MTx System, xSens Motion Technologies, http://www.xsens.com

[11] L. Cheng, S. Hailes, "An Experimental Study on a Motion Sensing System for Sports Training", appears as short paper in the Proceedings of the $5^{\text {th }}$ European Confernece on Wireless Sensor Networks (EWSN), Bologna, Italy, Feb 2008.

[12] K. Cocker, G. Cardon, I. Bourdeaudhuij, "Validity of the Inexpensive Stepping Meter in counting Steps in Free Living Conditions: a Pilot Study", in British Journal of Sports Medicine, 2006; 40:714-716.

[13] S. Nakazawa, T. Ishihara, H. Inooka, "Real-time Algorithms for Estimating Jerk Signals from Noisy Acceleration Data", in International Journal of Applied Electromagnetics and Mechanics, Vol. 18 (2003), pp. 149-163. 